\title{
Studying Whole-Mounted Sections of the Paranasal Sinuses to Understand the Complications of Endoscopic Sinus Surgery
}

Michael Rontal, MD; Eugene Rontal, MD

Endoscopic techniques for paranasal sinus surgery have allowed detailed and complete removal of sinus disease while promising minimum distress to the patient. The telescopic view of the operative field shows detail of the sinus anatomy and its disease, not possible in earlier transnasal techniques. Several articles document the serious complications seen with the endoscopic surgery. To understand the paranasal sinuses and their relationships to the orbit and cribriform plate, blocks of cadaver heads that included the orbit and paranasal sinuses were whole sectioned. It has been possible to see areas of the cribriform and orbital wall that are at risk to produce cerebrospinal fluid rhinorrhea and orbital complications. At the same time, landmarks for avoiding these complications can be defined to guide the surgeon during this dissection as seen through the endoscope.

\section{INTRODUCTION}

The introduction of endoscopes to paranasal sinus surgery has not eliminated the complications that occur with these operations. To fully understand how these complications are produced, a thorough and detailed understanding of the paranasal sinuses and their boundaries is needed. In the past, gross and gross-sectioned anatomical studies have been undertaken. ${ }^{1-6}$ Kirschner, et al. ${ }^{7}$ and Rontal, et al. ${ }^{8}$ have measured the distances between important structures of the medial orbital wall in an attempt to provide a quantitative description of this anatomy. These distances are reproduced here in Table I. This paper presents the findings from whole-sectioned and stained blocks that include the paranasal sinuses and orbits and correlate them with the complications of ethmoid/sphenoid surgery.

Presented at the Meeting of the Middle Section of the American Laryngological, Rhinological and Otological Society, Inc., Tulsa, January 21,1990 . This paper was presented in part as a Candidate's Thesis to the Society in 1980.

From the Department of Otolaryngology-Head and Neck Surgery, University of Michigan, Sinai Hospital of Detroit.

Send Reprint Requests to Michael Rontal, MD, 28500 Orchard Lake Road, Suite 200, Farmington Hills, MI 48018

\section{MATERIALS AND METHODS}

Six embalmed human cadaver heads were dissected to produce 12 blocks of tissue. Each block was fashioned to have as its boundaries the floor of the anterior cranial fossa, the skin of the face, and the malar bone. The sella turcica was cut to allow inclusion of the optic chiasm. The block from one side would include the nasal septum and the cribriform plate. The other side had only the turbinates as the medial border. The floor of the maxillary sinus was not included due to the size requirements for sectioning. Thus, the blocks encompassed the orbit with all of its walls and all of the paranasal sinuses. The cribriform plate/roof of the nose and the roof of the ethmoid were all included.

Each block was decalcified for 3 months, then cleared and stepped through dehydration until finally embedded in Epon. The blocks were sectioned as a unit on a Leitz ${ }^{\circledR}$ sliding microtome. The alignment was taken to match the standard orientation used in anatomy and in computer assisted tomography, i.e., the coronal, axial, and sagittal planes.

The sections produced were $15 \mu \mathrm{m}$ thick; they were serially mounted and stained (alternate sections) with hematoxylin-eosin and Masson trichrome stain (the latter differentiates muscle from fibrous tissue).

TABLE I.

Distance Between Key Orbital Structures.

\begin{tabular}{lrr}
\hline & $\begin{array}{c}\text { Mean } \\
(\mathrm{mm})\end{array}$ & $\begin{array}{r}\text { Range } \\
(\mathrm{mm})\end{array}$ \\
\hline $\begin{array}{l}\text { Anterior lacrimal crest to: } \\
\quad \text { Posterior lacrimal crest }\end{array}$ & 8 & $5-10$ \\
$\quad \begin{array}{l}\text { Anterior ethmoid artery } \\
\text { Posterior ethmoid artery }\end{array}$ & 24 & $20-28$ \\
$\quad$ Optic canal & 36 & $29-40$ \\
Frontoethmoid suture to: & 42 & $37-48$ \\
$\quad$ Maxilloethmoid suture & & \\
Posterior ethmoid foramen to: & 13 & $7-17$ \\
$\quad$ Anterior ethmoid foramen & & \\
$\quad$ Optic canal (anterior rim) & 11 & $5-16$ \\
$\begin{array}{l}\text { Infraorbital foramen to: } \\
\quad \text { Posterior wall of maxilla }\end{array}$ & 7 & $3-12$ \\
$\quad$ Optic canal & 36 & $26-44$ \\
Supraorbital notch to: & 48 & $40-54$ \\
$\quad$ Optic canal & & \\
\hline \hline
\end{tabular}



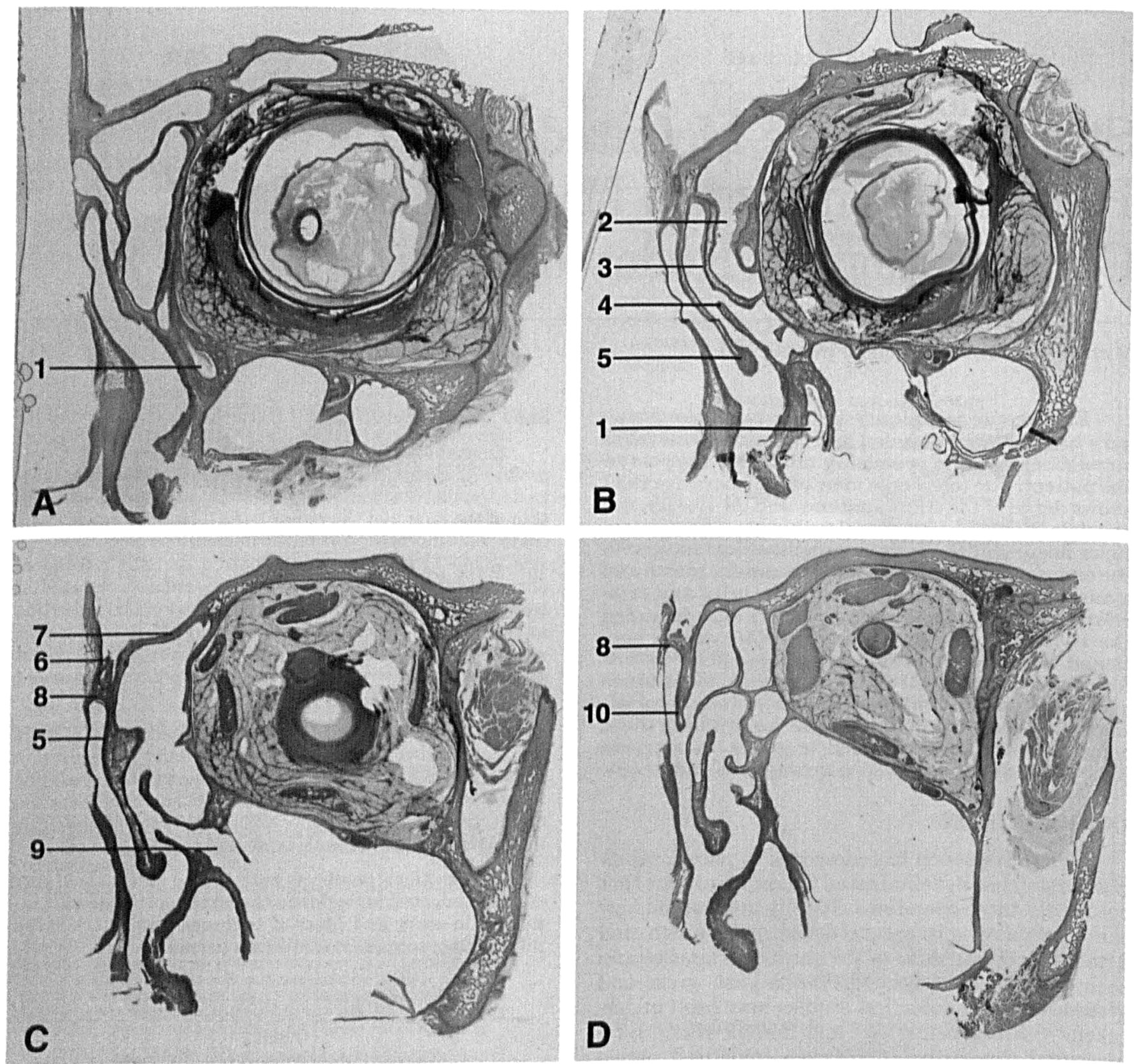

Figs. A-D. Coronal sections through the A. uncinate process; B. hiatus semilunaris anterior to the maxillary ostium; C. maxillary ostium; D. posterior ethmoid cells and anterior to the sphenoid face. $1=$ nasolacrimal duct; $2=$ frontal duct; $3=$ ethmoid bulla; $4=$ uncinate process; $5=$ middle turbinate; $6=$ olfactory nerve; $7=$ roof of ethmoid sinuses/floor of anterior cranial fossa; $8=$ cribriform plate; $9=$ ostium of maxillary sinus; $10=$ superior turbinate (H\&E, original magnification $\times 1.5)$.

The whole-mounted sections were studied directly and as photographs taken with transmitted light.

\section{RESULTS}

Figures A through $\mathrm{K}$ are reproduced here at $150 \%$ enlargement. The important structures can be arranged by the walls of the ethmoid complex, superior and lateral, as well as the sphenoid sinus posteriorly.

\section{Superior Ethmoid Wall or Roof}

The attachment of the perpendicular plate of the middle turbinate is the key structure of this wall. Medial to the turbinate is the thick cribriform plate, with the olfactory nerve fibers passing through (Figs. $\mathrm{B}, \mathrm{C})$. Lateral to the middle turbinate and the roof of the ethmoid complex is the plate of the frontal bone. This becomes thin as it extends laterally over ethmoid cells and the orbit. Note also that where the septae of the ethmoid cellular walls attach to the roof, the bone thickens but becomes thin between these septae. (Figs. C, I).

The roof itself projects above the orbit and above 

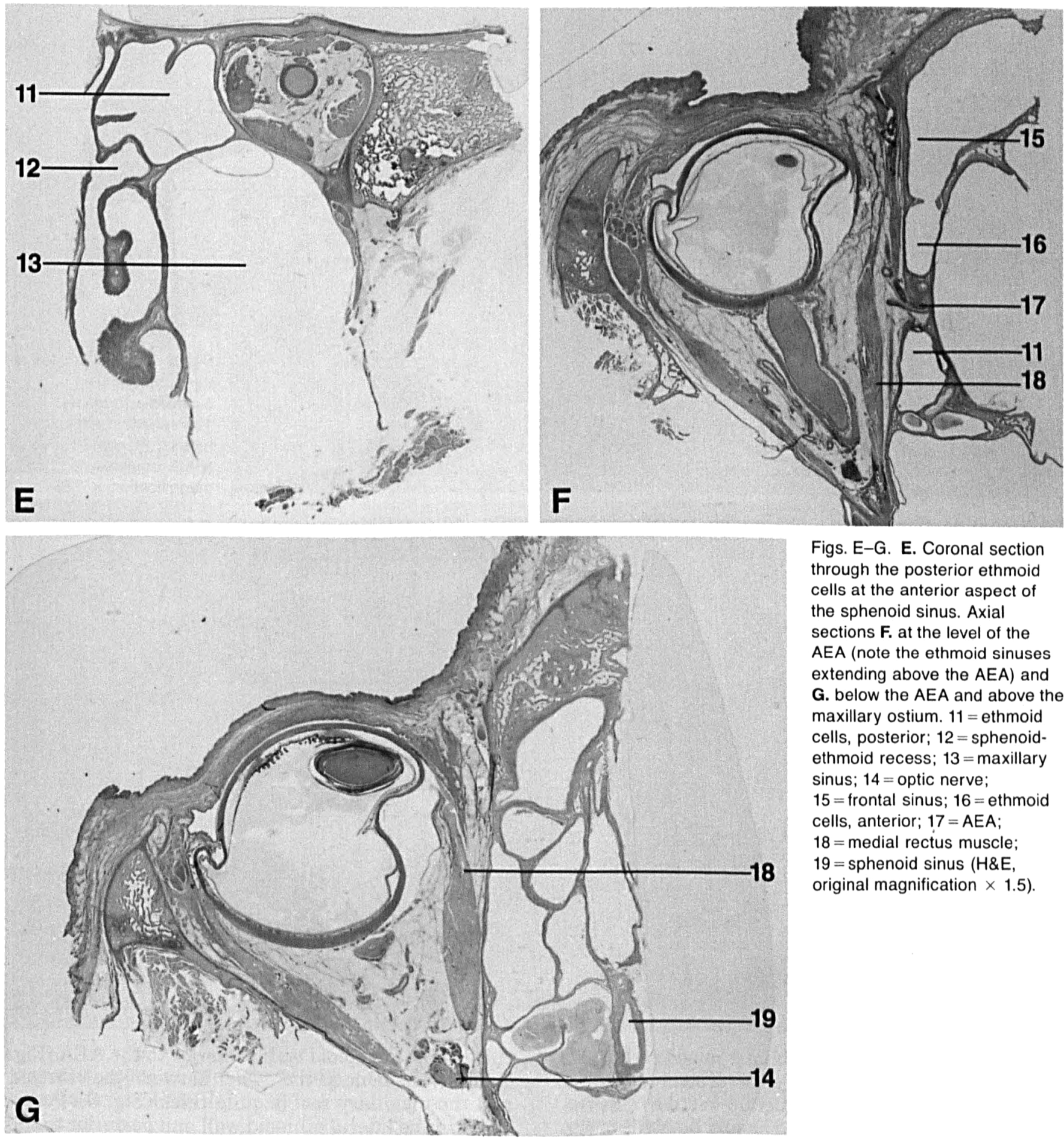

Figs. E-G. E. Coronal section through the posterior ethmoid cells at the anterior aspect of the sphenoid sinus. Axial sections $\mathbf{F}$. at the level of the AEA (note the ethmoid sinuses extending above the AEA) and G. below the AEA and above the maxillary ostium. $11=$ ethmoid cells, posterior; $12=$ sphenoidethmoid recess; $13=$ maxillary sinus; $14=$ optic nerve; $15=$ frontal sinus; $16=$ ethmoid cells, anterior; $17=\mathrm{AEA}$; $18=$ medial rectus muscle; $19=$ sphenoid sinus $(H \& E$ original magnification $\times 1.5)$

the anterior and posterior ethmoid arteries as the fovea ethmoidalis (Figs. C, F, K). The dura is closely applied to the roof of the ethmoid.

\section{Lateral Ethmoid Wall}

The key structures of this wall are the anterior ethmoid artery (AEA) and the attachment of the inferior turbinate. As it passes through the frontoethmoid suture, the AEA defines the superior extent of the medial wall. This artery also defines the placement of the natural ostium of the maxillary sinus that resides in the hiatus semilunaris as directly inferior from this artery (Fig. I). The horizontal level of the ostium is just above the inferior turbinate's attachment to the maxilla (Fig. C). The nasolacrimal duct is found anterior to the ostium of the maxillary sinus (Figs. A, B).

The AEA also defines the position of orbital structures. Thus, the globe is seen to be entirely anterior to the artery. The artery exits the orbit between the 

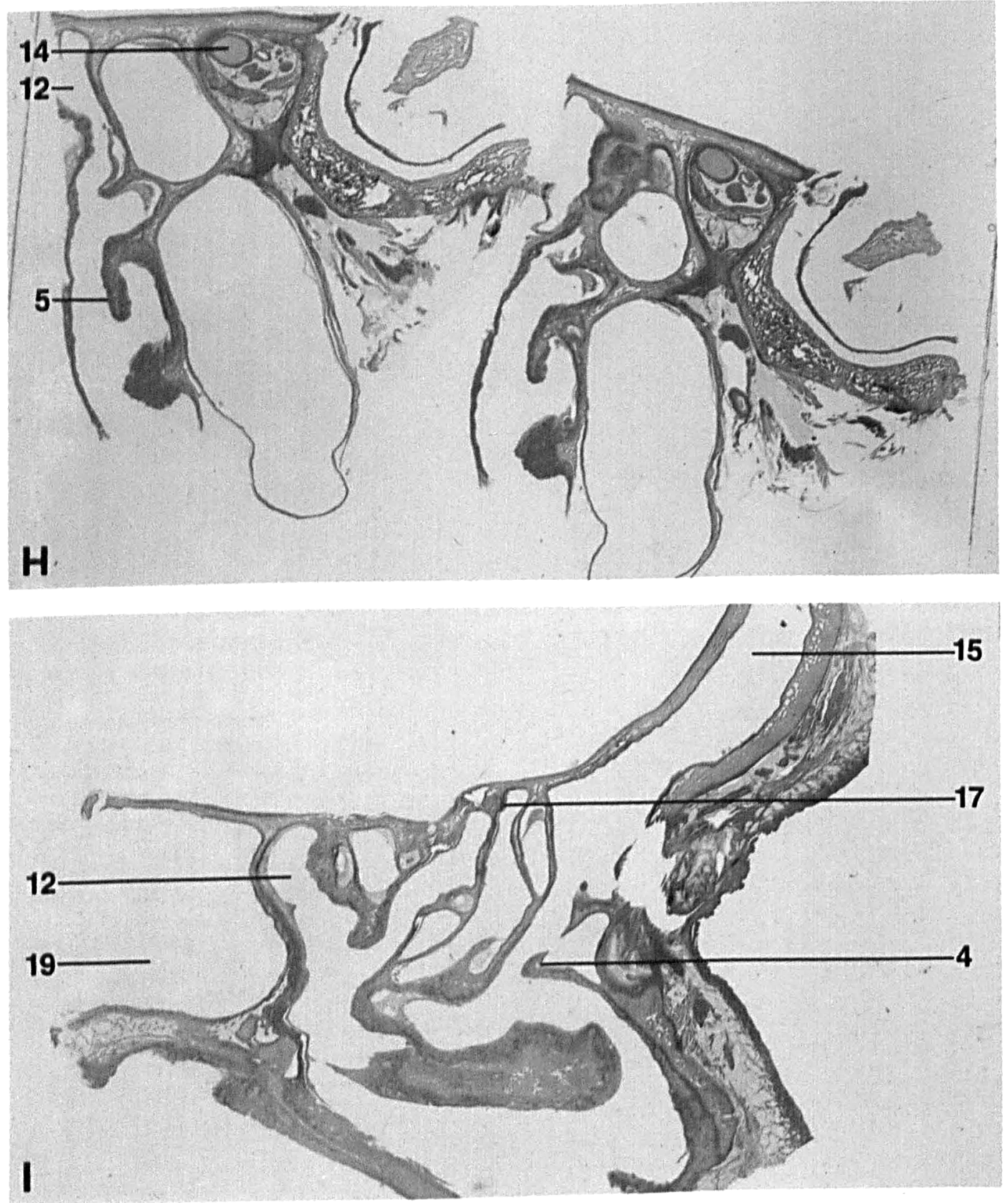

Fig. $\mathrm{H}$. Coronal section at the orbital apex. $5=$ middle turbinate; 12 = sphenoid-ethmoid recess; $14=$ optic nerve (H\&E, original magnification $\times 1.5$ ).
Fig. I. Parasagittal section through cribriform plate. $4=$ uncinate process; $12=$ sphenoid ethmoid recess; $15=$ frontal sinus; $17=$ anterior ethmoid artery; $19=$ sphenoid sinus ( $\mathrm{H} \& \mathrm{E}$, original magnification $\times 1.5$ ). medial rectus and superior oblique muscles (Fig. F). Anterior and below the artery the orbital fat pad is quite thick, separating the medial rectus from the periorbita (Figs. A-C, G). Note should be made of the paucity of vessels in the orbital fat and that those found are very small. There were no vessels seen closer than $4 \mathrm{~mm}$ to the lateral ethmoid wall. Posterior and inferior to this artery, the fat pad is thin and provides little safety during surgery (Figs. D-H).

It is seen from these slides that the thin lamina papyracea lies in a sagittal plane extending posteriorly from the inner canthus, where in practice it is palpable from the exterior (Figs. F, G). The maxillary sinus roof rises at a $30^{\circ}$ angle from anterior to posterior (Fig. K). It thus occupies the lower half to two thirds of the ethmoid wall posterior to the AEA (Fig. E). Also, the bone at the corner between the ethmoid and the maxillary roof is quite thick (Fig. C). Posteriorly in the lateral ethmoid wall and posterior to the anterior face of the sphenoid sinus, the optic nerve leaves the orbit at almost right angles to the plane of this wall (Fig. J).

\section{Sphenoid Sinus}

The posterior nasopharyngeal wall defines the plane just anterior to the exit of the optic nerve from the orbit (Figs. I-K). This is about equivalent to the anterior sphenoid wall. There are ethmoid cells that extend lateral and posterior to the sphenoid sinus and above it (Fig. G). Within the sphenoid sinus, the optic 

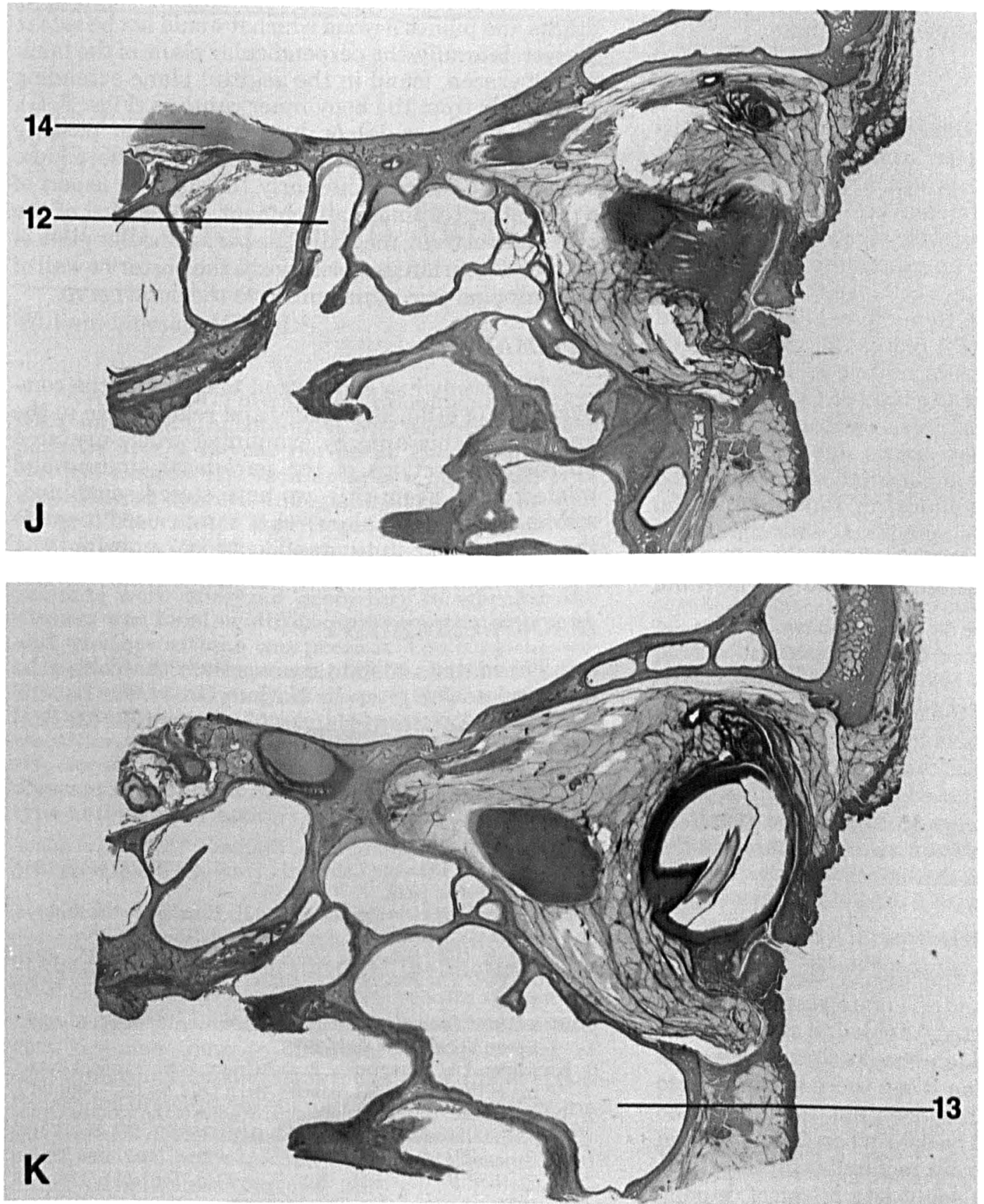

Fig. J. Parasagittal section through orbital canal. $12=$ sphenoidethmoid recess; $14=$ optic nerve $(H \& E$, original magnification $\times 1.5$ )
Fig. K. Parasagittal section through the sphenoid sinus (H\&E, original magnification $\times 1.5)$. nerve is seen on the lateral sinus wall above the carotid artery (Fig. J).

\section{DISCUSSION}

The anatomy seen in these sections is useful in correlating the complications of ethmoid/sphenoid surgery to the endoscopic techniques currently in use. To this end, the complications can be organized according to the wall on which they occur: superior, lateral, or posterior.

\section{Superior}

On the superior wall, cerebrospinal fluid rhinor- rhea is the most dangerous complication. The actual attachment of the middle turbinate and the intersinus septae may be thick, but the bone between may be thin. If torque is applied to the perpendicular plate of the middle turbinate or to the sinus septae right at the ethmoid roof, this thin plate could be fractured.

Medial to the middle turbinate, the thick cribriform plate is not easily fractured. The cribriform plate is a thick plate of bone. At the level of the superior turbinate the cribriform plate may be thin, though this is not an area of dissection. Rather, the aggressive removal of mucosa medial to the turbinate will injure the delicate olfactory fibers passing through the cribriform. There is little reason to dissect medial to the 
middle turbinate or the superior turbinate.

\section{Lateral}

Along the medial orbital wall, the complications of ethmoid surgery are expanding orbital hemorrhage, diplopia, ecchymosis with orbital emphysema, and tearing. Removal of medial orbital bone near the AEA may lacerate this vessel, allowing it to retract into the orbit and lead to an expanding orbital hematoma. This can enlarge and collapse the venous outflow from the optic nerve. Removal of lateral ethmoid wall at the level of the AEA places the medial rectus muscle and superior rectus muscle at risk, with a resulting diplopia. Below the level of the AEA, entry into the orbit can decompress orbital fat into the sinus, but little else. There are no significant vessels near the wall, though air and a small amount of blood may accumulate and produce an innocuous eyelid ecchymosis and orbital emphysema. Tearing can be produced by dissection through the thick bone anterior to the maxillary ostium and into the lacrimal duct.

\section{Posterior}

The posterior wall of the nasopharynx defines the anterior aspect of the sphenoid sinus, but also a plane anterior to which the optic nerve is not found. Thus, ethmoid cells that are followed posterior to this plane may expose the optic nerve to injury and result in blindness. The optic nerve can also be exposed in the sphenoid sinus as well as the carotid artery.

\section{Limits of Surgical Dissection}

We believe that by the use of the qualitative results presented here and the quantitative features we have previously reported, 8 Table I, it is possible to set a series of planes and landmarks that can be used to limit surgical dissection when there is doubt as to risking complication. This does not mean that no further dissection should be done when clear view and identification of the ethmoid roof and orbital walls is made. Rather, if there is doubt and a fear of causing cerebrospinal rhinorrhea or orbital hemorrhage or optic nerve trauma, it is possible to use certain land- marks and planes beyond which it would not be safe to dissect: laterally, the perpendicular plane of the lamina papyracea, found in the sagittal plane extending posteriorly from the bony inner canthus (Figs. F, G); superiorly, the axial or horizontal plane passing through the AEA and the attachment of the middle turbinate to the roof; inferiorly, the superior aspect of the inferior turbinate attachment or the level of the maxillary ostium; medially, the perpendicular plate of the middle turbinate; posteriorly, the posterior wall of the nasopharynx, which protects the optic nerve.

\section{SUMMARY}

This study has endeavored to correlate the complications of ethmoid surgery and relate these to the anatomy of this area by examining whole-mounted microscopic sections of the paranasal sinuses and related orbit. From this, we have stated some landmarks and surgical planes that can be used to guide the surgeon and, in times of doubt, serve as limits of dissection to help prevent surgical complications.

\section{ACKNOWLEDGMENT}

The authors wish to express their thanks for the technical advice given by Nathan Gross, $\mathrm{PhD}$ at the Kresge Ear Institute, University of Michigan Medical Center, Ann Arbor.

\section{BIBLIOGRAPHY}

1. Mosher, H.P.: Symposium on the Ethmoids: The Surgical Anatomy of the Ethmoid Labyrinth. Trans Am Acad Ophth Otol, 31:376-410, 1929.

2. Schaeffer, J.P.: Sinus Maxillaris and Its Relation to the Embryo, Child and Adult Man. Am J Anat, 10:313-368, 1910.

3. Van Alyea, O.E.: Sphenoid Sinus. Arch Otol, 34:225-253, 1941.

4. Ritter, F.N.: The Paranasal Sinuses. C.V. Mosby Co., St. Louis, 1973.

5. Rice, D. and Schaefer, S.: Endoscopic Paranasal Sinus Surgery. Raven Press, New York, 1988.

6. Kirschner, J.A., Yanagisawa, E. and Crelin, E.S.: Surgical Anatomy of the Ethmoidal Arteries. Arch Otol, 74:382-386, 1961.

7. Takahe shi, R.: Clinical Anatomical Studies of the Canalis Orbitocranialis and Canalis Orbitoethmoidalis in Relation to the Ethmoid Cells. In: A Collection of Ear, Nose and Throat Studies. R. Takahashi (Ed.). Kyoya Co., Tokyo, pp. 174-204, 1971.

8. Rontal, E., Rontal, M. and Guilford, F.T.: Surgical Anatomy of the Orbit. Ann Otol, 88:382-386, 1979. 